\title{
Questionário Hearing Handicap Inventory for the Elderly - Screening version (HHIE-S): estudo da sensibilidade e especificidade
}

\author{
Questionnaire Hearing Handicap Inventory for the Elderly - Screening \\ version (HHIE-S): sensitivity and specificity study
}

\author{
Ana Carolina Argondizo de Rosis¹, Marilia Rodrigues Freitas de Souza², Maria Cecília Martinelli Iório³
}

\begin{abstract}
RESUMO
Objetivo: Investigar a sensibilidade e especificidade do questionário Hearing Handicap Inventory for the Elderly - Screening version (HHIE-S) na triagem auditiva de idosos que procuram diferentes serviços de atenção à saúde (clínica audiológica e outras clínicas). Métodos: Foi avaliada a audição de 78 idosos - 23 atendidos na disciplina de Geriatria e Gerontologia da instituição de origem, que não tinham, necessariamente, queixas relacionadas à audição, e 55 atendidos no Ambulatório de Audiologia da mesma instituição, com queixas relacionadas aos sistemas auditivo e/ou vestibular. Foi aplicado o HHIE-S, cujo escore total foi dividido em três categorias, de acordo com a percepção do handicap. Resultados: Houve relação estatisticamente significante entre handicap e grau de perda auditiva no grupo de pacientes do Ambulatório de Audiologia $(\mathrm{p}=0,009)$, e no grupo de pacientes do Ambulatório de Geriatria $(\mathrm{p}=0,002)$. No primeiro grupo, o questionário HHIE-S revelou baixa sensibilidade $(23,5 \%)$ e alta especificidade $(73,7 \%)$. No grupo de pacientes do setor de Geriatria, foram altos os valores de sensibilidade (94,7\%) e de especificidade (75\%). Conclusões: Há associação positiva entre o grau de perda auditiva e o handicap referido em ambos os grupos. O questionário HHIE-S é um instrumento de triagem auditiva com alta especificidade e sensibilidade na identificação de perdas auditivas em idosos que procuram serviços de saúde que não são específicos para atendimentos relacionados às alterações auditivas.
\end{abstract}

Descritores: Transtornos da audição; Perda auditiva; Idoso; Audiometria; Questionários

\section{INTRODUÇÃO}

Todos os seres vivos estão sujeitos ao processo de envelhecimento, o qual é marcado pela diminuição do desempenho no meio em que vivem e da capacidade de adaptação ao mesmo. O ser humano é submetido ao processo de desenvolvimento até os 25 anos, aproximadamente, quando atinge o ápice de suas funções. Dessa faixa etária em diante, tal atividade dá lugar a uma série de alterações que representam o processo de envelhecimento, o qual é progressivo e degenerativo; universal; intrínseco, embora possa ser influenciado pelo meio; e distinto de doenças e patologias ${ }^{(1)}$.

São consideradas idosas, em países desenvolvidos, todas

Trabalho realizado no Departamento de Fonoaudiologia da Universidade Federal de São Paulo - UNIFESP - São Paulo (SP), Brasil.

(1) Aluna do Curso de Especialização em Audiologia Clínica da Irmandade da Santa Casa de Misericórdia de São Paulo - ISCSP - São Paulo (SP), Brasil. (2) Aluna do Curso de Especialização em Audiologia da Universidade Federal de São Paulo - UNIFESP - São Paulo (SP), Brasil.

(3) Livre-docente, Professora Associada do Departamento de Fonoaudiologia da Universidade Federal de São Paulo - UNIFESP - São Paulo (SP), Brasil. Endereço para correspondência: Ana Carolina Argondizo de Rosis. R. Maestro Cardim, 963/41, Paraíso, São Paulo (SP), Brasil, CEP: 01323-001. Email: nina_rosis@yahoo.com.br

Recebido em: 15/8/2008; Aceito em: 18/3/2009 as pessoas com idade superior a 65 anos; e em países em desenvolvimento todas aquelas com idade superior a $60 \operatorname{anos}^{(2)}$. O crescimento da população de idosos, em números absolutos e relativos, é um fenômeno mundial e está ocorrendo a um nível sem precedentes. De modo geral, vem se observando um crescimento da população idosa de forma mais acentuada nos países em desenvolvimento, embora este contingente ainda seja proporcionalmente inferior ao encontrado nos países desenvolvidos.

A análise dos dados referentes ao Censo de 2000 indica no Brasil uma população de idosos correspondendo a 8,6\% da população total (1.453.629 pessoas). Com base em projeções realizadas e considerando a continuidade das tendências verificadas para as taxas de fecundidade e longevidade da população brasileira, as estimativas para os próximos 20 anos indicam que a população idosa poderá exceder 30 milhões de pessoas, chegando a representar quase $13 \%$ da população ${ }^{(3)}$.

Sabe-se que o aumento da idade cronológica é responsável por mudanças morfológicas e funcionais que atingem todo o organismo. São acometidos, dentre outros, os sistemas: nervoso, endócrino, imunológico, cardiovascular, respiratório, hemopoiético, digestivo, geniturinário e musculo-esquelético. Além disso, são evidentes alterações da pele, do peso corporal, bem como dos órgãos do sentido, os quais se tornam menos 
eficientes, interferindo na segurança, nas atividades diárias e no bem-estar geral dos indivíduos. Dentre os sentidos mais afetados, está a audição, que vem a ser o objeto de investigação desse estudo.

A presbiacusia é uma perda auditiva sensorioneural bilateral, simétrica, que compromete mais as frequiências altas e cuja origem deve-se apenas ao envelhecimento.

A degeneração que mais marcadamente interfere com a audição envolve as estruturas da orelha interna ${ }^{(4)}$. A presbiacusia é classificada em quatro categorias: (1) sensorial - marcada pela atrofia do órgão de Corti, perda de células ciliadas e de sustentação; (2) neural - com perda de fibras nervosas ou células do sistema nervoso central; (3) metabólica - marcada pela atrofia da estria vascular e desequilíbrio bioelétrico/bioquímico da cóclea; (4) mecânica - com alterações no movimento do duto coclear e da membrana basilar ${ }^{(5)}$.

A presbiacusia também é definida como uma deficiência na sensibilidade liminar auditiva e no reconhecimento de fala. Tais fatores comprometem o desempenho de idosos no processo de comunicação verbal ${ }^{(6)}$.

A deficiência auditiva gera no idoso um dos mais incapacitantes distúrbios de comunicação, impedindo-o de desempenhar plenamente seu papel na sociedade. Ser um idoso com deficiência auditiva adquirida traz implicações sociais sérias para a vida desse indivíduo e para os que com ele convivem ${ }^{(7)}$. Há duas importantes consequiências da deficiência auditiva: a incapacidade auditiva, que se refere a qualquer limitação ou falta de habilidade para desempenhar uma atividade devido aos problemas auditivos vivenciados pelo indivíduo com perda; e o handicap (desvantagem), que diz respeito aos aspectos nãoauditivos resultantes da deficiência e da incapacidade auditivas, os quais restringem a participação do idoso nas atividades de vida diária e comprometem suas relações sociais ${ }^{(2)}$.

A indicação e adaptação de próteses auditivas ou Aparelhos de Amplificação Sonora Individuais - AASI - podem minimizar os efeitos negativos da deficiência auditiva nessa população. Porém, devemos considerar que exames audiométricos que possibilitem a adaptação de tais instrumentos são responsáveis por fornecer apenas informações básicas sobre as habilidades auditivas do indivíduo, limitando-se ao grau e tipo da perda auditiva. Sabe-se, no entanto, que é comum encontrar uma perda significativa da sensibilidade por freqüência e pouca queixa em relação ao uso funcional da audição, bem como é possível encontrar o contrário: idosos com perda auditiva leve podem apresentar alto índice de percepção de desvantagem funcional ${ }^{(8)}$. Assim, é imprescindível que se avalie de que modo a perda auditiva afeta os aspectos emocionais e sociais de seu portador; o que pode ser realizado por meio da aplicação de questionários de auto-avaliação, os quais permitem uma visão mais completa do perfil audiológico de cada indivíduo e da percepção de seu problema, levando-o a obter uma maior qualidade de vida por meio de um trabalho de reabilitação mais personalizado e específico.

Um exemplo desse tipo de instrumento é o Hearing Handicap Inventory for the Elderly Screening Version - HHIE-S ${ }^{(9)}$. Trata-se de uma versão reduzida do Hearing Handicap Inventory for the Elderly - HHIE, de aplicação mais rápida e de fácil compreensão, o que favorece seu uso em indivíduos idosos, sendo por isso recomendado pela American Speech-LanguageHearing Association (ASHA) para triagem auditiva ${ }^{(10)}$.

Com base no que o questionário Hearing Handicap Inventory for the Elderly Screening Version - HHIE-S pretende avaliar, o presente trabalho teve como objetivo investigar sua sensibilidade e especificidade na triagem auditiva de idosos que procuram diferentes serviços de atenção à saúde (clínica audiológica e outras clínicas).

\section{MÉTODOS}

A execução do trabalho foi aprovada pelo Comitê de Ética em Pesquisa da Universidade Federal de São Paulo (UNIFESP), por meio do protocolo de número 1479/06, de 05 de outubro de 2006.

A coleta de dados foi realizada no período compreendido entre outubro de 2006 e outubro de 2007. A amostra foi formada por pacientes da Disciplina de Geriatria e Gerontologia e do Ambulatório de Audiologia da instituição de origem.

Para compor a amostra, os pacientes obedeceram aos seguintes critérios de inclusão: ter mais de 60 anos, não ser protetizado e ter um nível de compreensão oral que permitisse a aplicação do questionário. Inicialmente, os pacientes assinaram o Termo de Consentimento Livre e Esclarecido, autorizando o uso dos dados coletados no estudo, de acordo com as normas estabelecidas pelo Comitê de Ética da instituição.

Foi, então, avaliada a audição de 78 idosos, por meio da audiometria tonal liminar (freqüências de 250 a $8000 \mathrm{~Hz}$ para via aérea e de 500 a $4000 \mathrm{~Hz}$ para via óssea, esta última realizada apenas nos casos em que os limiares auditivos das frequiências avaliadas por via aérea fossem iguais ou maiores que $25 \mathrm{~dB}$ NA) e da logoaudiometria (SRT - Limiar de Recepção de Fala; e IPRF - Índice Percentual de Reconhecimento de Fala), com uso do audiômetro modelo MA-41 e fone TDH-39, em cabina acústica. A Imitanciometria foi feita com o auxílio do imitanciômetro da marca Interacoustics ${ }^{\circledR}$, modelo AZ7, e constou da timpanometria e da pesquisa dos limiares dos reflexos acústicos contralaterais nas frequiências de 500, 1000, 2000 e $4000 \mathrm{~Hz}$.

Os idosos foram distribuídos em grupos, de acordo com os diferentes graus de perda auditiva, determinados com base na média dos limiares obtidos nas freqüências de 500, 1000 e $2000 \mathrm{~Hz}$ na melhor orelha ${ }^{(11)}$, e com o local de procedência do paciente. Assim, fizeram parte do estudo 23 indivíduos atendidos na Disciplina de Geriatria e Gerontologia da UNIFESP, sendo $18(78,3 \%)$ do sexo feminino e $5(21,7 \%)$ do sexo masculino, que não tinham, necessariamente, queixas relacionadas à audição; e 55 indivíduos atendidos no Ambulatório de Audiologia da UNIFESP, sendo 38 (69,1\%) do sexo feminino e $17(30,9 \%)$ do sexo masculino, que possuíam queixa relacionada aos sistemas auditivo e/ou vestibular. Cada um desses grupos foi dividido em três subgrupos, de acordo com os limiares auditivos encontrados: pacientes com limiares auditivos normais (média até $25 \mathrm{~dB}$ NA), pacientes com perda auditiva de grau leve (média entre 26 e $40 \mathrm{~dB}$ NA) e pacientes com perda de grau moderado ou maior (média acima de 41 dB NA).

Foi, então, aplicado o questionário Hearing Handicap 
Inventory for the Elderly Screening Version - HHIE-S (Anexo 1), composto por dez perguntas, dividido em duas escalas (escala social/situacional e escala emocional, cada uma com cinco itens). Para responder ao questionário, foram lidas oralmente as perguntas ao participante, que deveria optar por apenas uma resposta para cada item: $\operatorname{sim}$ (4 pontos), algumas vezes ( 2 pontos) ou não ( 0 ponto). $\mathrm{O}$ escore total variou de 0 a 40, dividido em três categorias: $0-8$ pontos (sem percepção do handicap); 10-23 pontos (percepção leve a moderada) e 24-40 (percepção significativa do handicap).

Ao final, foi efetuada a análise estatística dos dados obtidos. A restrição de participação em atividades de vida diária, mensurada com o uso do questionário, foi relacionada aos graus de perda auditiva dos sujeitos pertencentes aos dois grupos estudados: pacientes atendidos na Disciplina de Geriatria e Gerontologia da UNIFESP e pacientes atendidos no Ambulatório de Audiologia da instituição. Vale registrar que foi denominada de handicap a restrição de participação em atividades de vida diária devido ao emprego desta expressão no questionário utilizado no presente estudo.

Foram empregados os testes de Qui-quadrado para Independência, a fim de verificar a associação existente entre o grau de perda auditiva e o grau do handicap. Foi feita a verificação da sensibilidade e especificidade para o uso do questionário HHIE-S como instrumento de triagem, considerando que a precisão de um teste em identificar corretamente os pacientes positivos (com distúrbio) é chamada sensibilidade e sua precisão em classificar corretamente os pacientes negativos (sem distúrbio) é chamada especificidade.

Para este estudo, foi adotado um nível de significância de $0,05(5 \%)$. Todos os intervalos de confiança obtidos ao longo do trabalho foram construídos com $95 \%$ de confiança estatística.

\section{RESULTADOS}

Dos 55 pacientes atendidos no Ambulatório de Audiologia, 17 (30,9\%) não apresentaram perda auditiva, 17 (30,9\%) apresentaram perda auditiva de grau leve e $21(38,2 \%)$ apresentaram perda auditiva de grau moderado ou maior. Já dos 23 pacientes atendidos no Setor de Geriatria, $19(82,6 \%)$ tinham audição normal, $2(8,7 \%)$ apresentaram perda auditiva de grau leve e $2(8,7 \%)$ apresentaram perda auditiva de grau moderado ou maior (Tabela 1 e Figura 1).

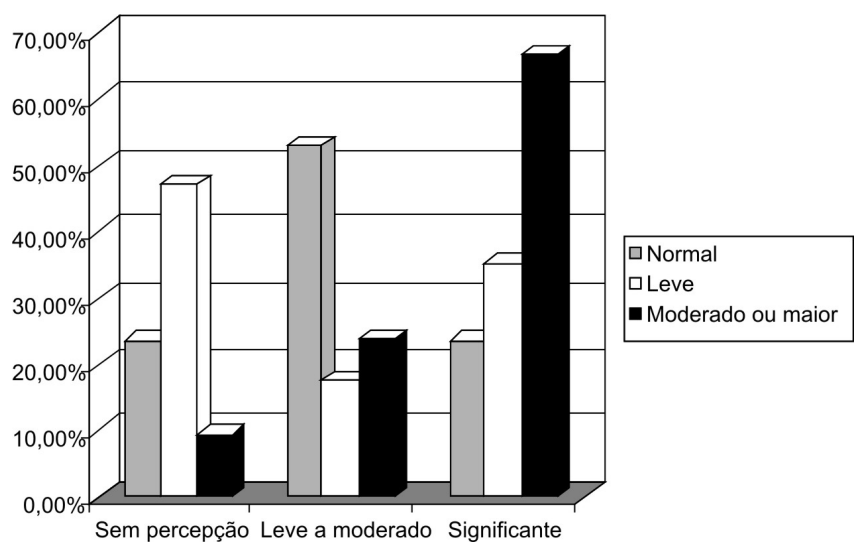

Figura 1. Associação entre handicap e grau da perda auditiva para o grupo de pacientes atendidos no Ambulatório de Audiologia

Para verificar se existia ou não uma associação entre handicap e grau de perda auditiva, foram estudados os valores em percentuais. Assim, comparou-se a distribuição da coluna total com a distribuição das demais colunas (intermediárias). Caso existisse algum valor por linha que estivesse muito diferente do valor da coluna total, isso seria sinal de uma provável associação e/ou dependência estatística.

Observou-se que existe relação e/ou associação estatisticamente significante entre restrição de participação em atividades de vida diária e grau de perda, ou seja, a pontuação obtida na aplicação do questionário está associada ao grau de perda. Para facilitar foram assinaladas com um asterisco (Tabela 1) as combinações nas quais a relação foi mais forte estatisticamente.

Vale notar que devido à baixa quantidade de respostas para os seguintes graus de perda: moderadamente severo, severo e profundo, tais classificações foram agrupadas em uma única categoria, denominada "moderado ou maior".

A mesma análise foi realizada também no grupo de pacientes atendidos no Ambulatório de Geriatria (Tabela 2 e Figura 2).

Notou-se que também no grupo de Geriatria existe relação e/ou associação estatisticamente significante entre handicap e grau de perda auditiva. Novamente, para facilitar e visualização das relações, foram assinaladas com um asterisco as combinações mais fortes.

Para o cálculo dos valores de sensibilidade e especificidade é preciso que se tenha uma tabela de quadrada (mesma quantidade de linhas e colunas) de tamanho $2 \times 2$, ou seja, com no máximo duas categorias de resposta para cada variável.

Tabela 1. Associação entre handicap e grau da perda auditiva para o grupo de pacientes atendidos no Ambulatório de Audiologia Handicap Audiometria

\begin{tabular}{|c|c|c|c|c|c|c|c|c|}
\hline & \multicolumn{2}{|c|}{ Normal } & \multicolumn{2}{|c|}{ Perda de grau leve } & \multicolumn{2}{|c|}{$\begin{array}{c}\text { Perda de grau moderado } \\
\text { ou maior }\end{array}$} & \multicolumn{2}{|c|}{ Total } \\
\hline & $\mathrm{n}$ & $\%$ & $\mathrm{n}$ & $\%$ & $\mathrm{n}$ & $\%$ & $\mathrm{n}$ & $\%$ \\
\hline Sem percepção & 4 & $23,5 \%$ & 8 & $47,1 \%{ }^{*}$ & 2 & $9,5 \%$ & 14 & $25,5 \%$ \\
\hline Leve a moderado & 9 & $52,9 \% *$ & 3 & $17,6 \%$ & 5 & $23,8 \%$ & 17 & $30,9 \%$ \\
\hline Significante & 4 & $23,5 \%$ & 6 & $35,3 \%$ & 14 & $66,7 \% *$ & 24 & $43,6 \%$ \\
\hline Total & 17 & $30,9 \%$ & 17 & $30,9 \%$ & 21 & $38,2 \%$ & 55 & $100 \%$ \\
\hline
\end{tabular}

Valor de $p=0,009$

Legenda: $\mathrm{n}=$ valor absoluto; \% = porcentagem; * combinações nas quais a relação foi mais forte estatisticamente 
Tabela 2. Associação entre handicap e grau da perda auditiva para o grupo de pacientes atendidos no Ambulatório de Geriatria

\begin{tabular}{|c|c|c|c|c|c|c|c|c|}
\hline \multirow[t]{3}{*}{ Handicap } & \multicolumn{8}{|c|}{ Audiometria } \\
\hline & \multicolumn{2}{|c|}{ Normal } & \multicolumn{2}{|c|}{ Perda de grau leve } & \multicolumn{2}{|c|}{$\begin{array}{c}\text { Perda de grau moderado } \\
\text { ou maior } \\
\end{array}$} & \multicolumn{2}{|c|}{ Total } \\
\hline & $\mathrm{n}$ & $\%$ & $\mathrm{n}$ & $\%$ & $\mathrm{n}$ & $\%$ & Qtde & $\%$ \\
\hline Sem Percepção & 18 & $94,7 \% *$ & 1 & $50,0 \%$ & 0 & $0,0 \%$ & 19 & $82,6 \%$ \\
\hline Leve a moderada & 1 & $5,3 \%$ & 1 & $50,0 \%$ & 2 & $100,0 \% *$ & 4 & $17,4 \%$ \\
\hline Total & 19 & $82,6 \%$ & 2 & $8,7 \%$ & 2 & $8,7 \%$ & 23 & $100 \%$ \\
\hline
\end{tabular}

Valor de $\mathrm{p}=0,002$

Legenda: $\mathrm{n}=$ valor absoluto; \% = porcentagem; * combinações nas quais a relação foi mais forte estatisticamente

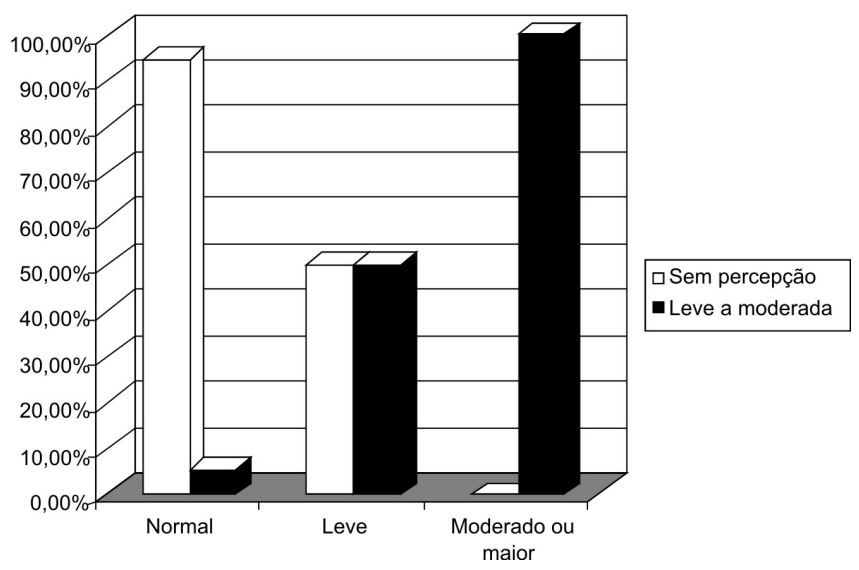

Figura 2. Associação entre handicap e grau da perda auditiva para o grupo de pacientes atendidos no Ambulatório de Geriatria

Sendo assim, as tabelas anteriores foram ajustadas e o grau de perda foi classificado em: normal e alterado (qualquer classificação diferente da normal) e o handicap ficou com a seguinte classificação: sem percepção e com percepção (qualquer classificação diferente da "sem percepção").

Desta forma, são apresentados os resultados da relação e/ou associação entre handicap e existência ou não de perda auditiva para o grupo de pacientes atendidos no Ambulatório de Audiologia da instituição (Tabela 3 e Figura 3), juntamente com os valores de sensibilidade e especificidade (Tabela 4).

Tabela 3. Associação entre o handicap e o resultado obtido na audiometria para o grupo de pacientes atendidos no Ambulatório de Audiologia

\begin{tabular}{lcccccc}
\hline Handicap & \multicolumn{6}{c}{ Audiometria } \\
\cline { 2 - 8 } & \multicolumn{2}{c}{ Normal } & \multicolumn{2}{c}{ Alterada } & \multicolumn{2}{c}{ Total } \\
\cline { 2 - 7 } & $\mathrm{n}$ & $\%$ & $\mathrm{n}$ & $\%$ & $\mathrm{n}$ & $\%$ \\
\hline Sem percepção & 4 & $23,5 \%$ & 10 & $26,3 \%$ & 14 & $25,5 \%$ \\
Com percepção & 13 & $76,5 \%$ & 28 & $73,7 \%$ & 41 & $74,5 \%$ \\
\hline Total & 17 & $30,9 \%$ & 38 & $69,1 \%$ & 55 & $100 \%$ \\
\hline
\end{tabular}

Valor de $\mathrm{p}=0,826$

Legenda: $\mathrm{n}=$ valor absoluto; $\%$ = porcentagem

Com as modificações, não houve relação e/ou associação estatisticamente significante entre handicap e presença de perda auditiva no grupo do Ambulatório de Audiologia. Verifica-se, também, que a sensibilidade é baixa e a soma de sensibilidade e especificidade é quase que $100 \%$, o que demonstra que os dados são quase casuais.

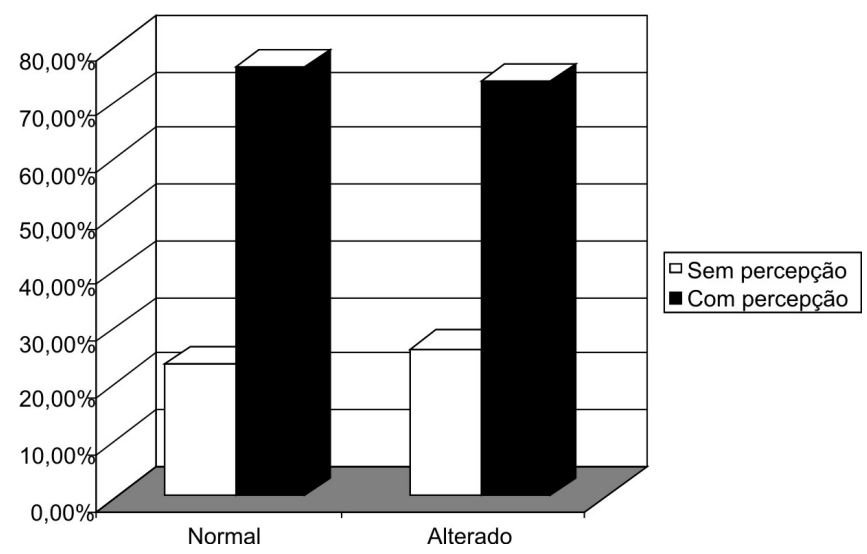

Figura 3. Associação entre o handicap e o resultado obtido na audiometria para o grupo de pacientes atendidos no Ambulatório de Audiologia

Tabela 4. Sensibilidade e especificidade do questionário HHIE-S para o grupo de pacientes atendidos no Ambulatório de Audiologia

\begin{tabular}{lcc}
\hline Estatistica & Resultado & IC \\
\hline Acurácia & $58,2 \%$ & $13,0 \%$ \\
Sensibilidade & $23,5 \%$ & $20,2 \%$ \\
Especificidade & $73,7 \%$ & $14,0 \%$ \\
VP+ & $28,6 \%$ & $23,7 \%$ \\
VP- & $68,3 \%$ & $14,2 \%$ \\
\hline
\end{tabular}

Legenda: $\mathrm{VP}+=$ valor preditivo positivo; $\mathrm{VP}-=$ valor preditivo negativo; $\mathrm{IC}=$ intervalo de confiança

Foi realizada a mesma análise no grupo de pacientes atendidos no Ambulatório de Geriatria da instituição de origem (Tabelas 5 e 6, e Figura 4).

Tabela 5. Associação entre o handicap e o resultado obtido na audiometria para o grupo de pacientes atendidos no Ambulatório de Geriatria

\begin{tabular}{lcccccc}
\hline Handicap & \multicolumn{7}{c}{ Audiometria } \\
\cline { 2 - 7 } & \multicolumn{2}{c}{ Normal } & \multicolumn{2}{c}{ Alterada } & \multicolumn{2}{c}{ Total } \\
\cline { 2 - 7 } & $\mathrm{n}$ & $\%$ & $\mathrm{n}$ & $\%$ & $\mathrm{n}$ & $\%$ \\
\hline Sem percepção & 18 & $94,7 \%$ & 1 & $25,0 \%$ & 19 & $82,6 \%$ \\
Com percepção & 1 & $5,3 \%$ & 3 & $75,0 \%$ & 4 & $17,4 \%$ \\
\hline Total & 19 & $82,6 \%$ & 4 & $17,4 \%$ & 23 & $100 \%$
\end{tabular}

Valor de $p=0,001$

Legenda: $\mathrm{n}=$ valor absoluto; \% = porcentagem; ${ }^{*}$ combinações nas quais a relação foi mais forte estatisticamente 
Tabela 6. Sensibilidade e especificidade do questionário HHIE-S para o grupo de pacientes atendidos no Ambulatório de Geriatria

\begin{tabular}{lcc}
\hline Estatística & Resultado & IC \\
\hline Acurácia & $91,3 \%$ & $11,5 \%$ \\
Sensibilidade & $94,7 \%$ & $10,0 \%$ \\
Especificidade & $75,0 \%$ & $42,4 \%$ \\
VP+ & $94,7 \%$ & $10,0 \%$ \\
VP- & $75,0 \%$ & $42,4 \%$ \\
\hline
\end{tabular}

Legenda: $\mathrm{VP}+=$ valor preditivo positivo; $\mathrm{VP}-$ = valor preditivo negativo; IC = intervalo de confiança

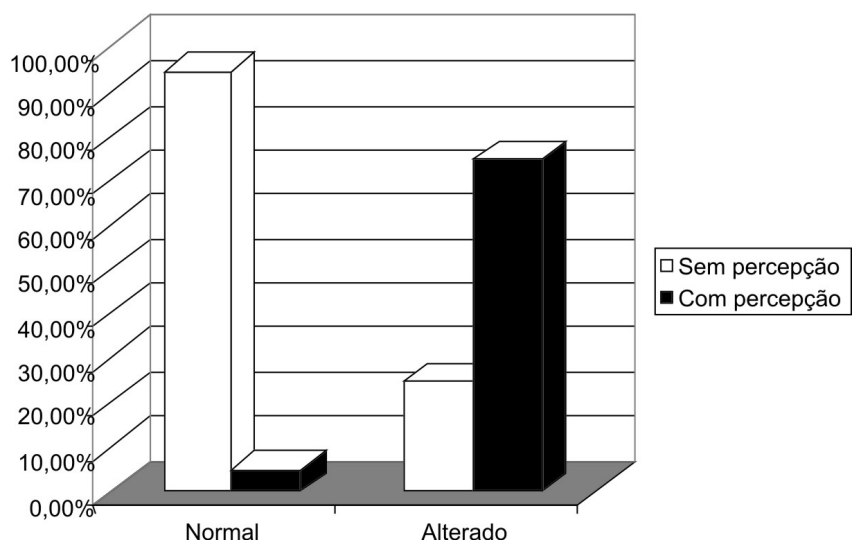

Figura 4. Associação entre o handicap e o resultado obtido na audiometria para grupo de pacientes atendidos no Ambulatório de Geriatria

Foi possível verificar que no grupo de pacientes atendidos no Setor de Geriatria, sem queixas relacionadas aos sistemas auditivo e/ou vestibular, existiu associação estatística entre os resultados obtidos na aplicação do questionário HHIE-S com a existência ou não de perda auditiva. Para facilitar, foram assinaladas com um asterisco as combinações mais evidentes estatisticamente. No quadro com os demais resultados, pode-se notar valores altos para especificidade e principalmente para sensibilidade.

\section{DISCUSSÃO}

O presente estudo mostra que tanto no grupo de pacientes atendidos no setor de Audiologia - com queixa auditiva (Tabela 1) quanto no grupo de pacientes atendidos no setor de Geriatria - que não apresentavam, obrigatoriamente, queixa auditiva (Tabela 2), é encontrada associação estatisticamente significante ao se comparar o grau de perda auditiva com o grau de handicap obtido na aplicação do questionário HHIE-S. Este achado corresponde a outro estudo, no qual foi encontrada correlação entre a severidade da perda auditiva e o número de pacientes que apresentavam handicap ao HHIE-S, embora muitos pacientes com perda auditiva não tenham referido handicap $^{(12)}$. Os resultados do presente estudo, no entanto, diferem dos resultados encontrados em outra pesquisa, a qual, após realizar um estudo da auto-percepção do handicap auditivo em idosos por meio do questionário HHIE-S em 40 indivíduos, concluiu que a percepção do prejuízo auditivo não estava relacionada ao grau da perda auditiva ${ }^{(13)}$.

No grupo de pacientes atendidos no Setor de Audiologia da instituição de origem, dos 17 pacientes normais, 4 (23,5\%) não referiram handicap. Dos 17 indivíduos com perda leve, $3(17,6 \%)$ referiram handicap leve a moderado e dos 21 com perda moderada ou maior, $14(66,7 \%)$ apresentaram percepção significante do handicap. Estes dados estão de acordo com o um estudo onde a prevalência do handicap foi maior nos indivíduos com perdas auditivas mais acentuadas, no qual relataram handicap $8 \%$ dos indivíduos sem perda, 29\% dos indivíduos com perda leve e $64,6 \%$ dos indivíduos com perda moderada/severa ${ }^{(14)}$.

Ainda no grupo de pacientes atendidos no Setor de Audiologia da instituição de origem, 2 (9,5\%), os pacientes sem auto-percepção do handicap apresentaram perda moderada ou maior e $66,7 \%$ dos pacientes com percepção significativa apresentaram perda também de grau moderado ou maior. Assim, houve variabilidade de resposta no questionário HHIE-S em idosos com a mesma sensibilidade auditiva, da mesma forma que em outra pesquisa realizada, na qual 7,4\% dos idosos sem auto-percepção do handicap apresentaram perda e $85 \%$ dos idosos com percepção significativa apresentaram perda auditiva $^{(15)}$.

No grupo de pacientes atendidos no Setor de Geriatria da instituição de origem, dos 19 pacientes normais, 18 (94,7\%) não referiram handicap; dos 2 pacientes com perda leve, 1 $(50,0 \%)$ revelou percepção leve a moderada do handicap e, dos 2 com perda de grau moderado ou maior, 2 (100,0\%) apresentaram percepção também leve a moderada do handicap. Os achados assemelham-se aos encontrados em estudo no qual $83 \%$ dos indivíduos sem perda (média abaixo de 25 dB NA) não referiram handicap e $70 \%$ dos indivíduos com média acima de $55 \mathrm{~dB}$ NA perceberam o handicap. Dos indivíduos com média dos limiares situada entre 26 e 40 dB NA, $44 \%$ não referiram handicap e 56\% referiram handicap leve a significante $^{(16)}$.

No que se refere à sensibilidade e especificidade do uso do questionário na triagem de idosos, pode-se afirmar, com base na análise estatística do presente trabalho, que em populações que já chegam ao serviço ambulatorial com queixas relacionadas à audição, o questionário não parece ser um bom instrumento de triagem, tendo em vista os baixos valores de sensibilidade $(23,5 \%)$ (Tabela 4). Estes dados são semelhantes aos já relatados na literatura, nos quais o uso do escore maior que 8 no HHIE-S como forma de triagem de idosos com deficiência auditiva também resultou em baixa sensibilidade $(34 \%)^{(17)}$. Da mesma forma, em estudo com 566 indivíduos idosos com história de exposição prévia ao ruído, o questionário HHIE-S revelou baixa sensibilidade (36\%) na triagem auditiva $^{(18)}$. Isso possivelmente ocorra pois a população que procura o serviço de avaliação audiológica após ser encaminhada por alguma especialidade médica, já chega a este local sensibilizada acreditando que, pelo fato de terem sido orientados a passar por uma investigação específica, possuam algum tipo de alteração da audição.

No entanto, pode-se dizer que o questionário HHIE-S é um bom instrumento para ser usado em populações que não tenham, obrigatoriamente, queixas relacionadas à audição, como é o caso da população atendida no Ambulatório de Geriatria da instituição de origem, onde o questionário re- 
velou maior sensibilidade $(94,7 \%)$ e especificidade $(75,0 \%)$ (Tabela 6). Assim, em populações sem queixa auditiva, o questionário parece ser eficiente tanto para verificar a proporção de indivíduos que realmente tenham uma alteração da audição (sensibilidade) quanto para verificar, em meio aos sem queixa, aqueles que, de fato, são audiologicamente normais (especificidade). No entanto, em um estudo realizado com 60 idosos não vinculados a programas de reabilitação auditiva, a fim de verificar a correlação entre os resultados obtidos no questionário HHIE-S com a presença ou ausência de perda auditiva segundo a audiometria, o questionário revelou alta sensibilidade $(84,4 \%)$ e baixa especificidade $(46,7 \%) \mathrm{em}$ detectar alterações da audição ${ }^{(19)}$.

Os dados do presente estudo são compatíveis com os descritos por autores que admitem o questionário HHIE-S como um bom teste de identificação de idosos com perda auditiva ${ }^{(20-21)}$. Outros autores também afirmaram que o HHIE-S é um instrumento de triagem que consegue identificar a maioria dos idosos com perdas auditivas em freqüências altas ${ }^{(22)}$.

É possível verificar, ainda, que no grupo de pacientes atendidos no Ambulatório de Audiologia, o questionário apresentou baixos valores preditivos: positivo $(28,6 \%)$ e negativo $(68,3 \%)$, enquanto que, no grupo de pacientes atendidos no Ambulatório de Geriatria, os valores preditivos positivo e negativo foram altos $(94,7 \%$ e $75,0 \%$, respectivamente). Isso mostra que, na população proveniente do Ambulatório de Geriatria, a probabilidade de se ter alguma alteração da audição é grande, caso seja alta a pontuação obtida no questionário HHIE-S, bem como é grande a probabilidade de não se ter nenhuma alteração da audição quando a pontuação do mesmo questionário for baixa. Isso, no entanto, não pode ser afirmado com relação à população atendida no Ambulatório de Audiologia da instituição de origem.

\section{CONCLUSÕES}

Há associação positiva entre o grau de perda auditiva e o grau de handicap referido pelos pacientes, tanto em idosos que procuram a clínica audiológica como naqueles que procuram outros serviços de atenção à saúde.

O questionário HHIE-S é um instrumento de triagem auditiva com alta especificidade e sensibilidade na identificação de perdas auditivas em idosos que procuram serviços de saúde que não são específicos para atendimentos relacionados às alterações da audição.

O questionário HHIE-S não é um instrumento eficiente (baixa sensibilidade) na triagem auditiva de idosos que procuram ou são encaminhados para a clínica audiológica.

\section{AGRADECIMENTOS}

À Fundação de Amparo à Pesquisa do Estado de São Paulo (FAPESP), pelo apoio concedido em forma de bolsa de Iniciação Científica.

\begin{abstract}
Purpose: To investigate the sensitivity and specificity of the questionnaire Hearing Handicap Inventory for the Elderly - Screening version (HHIE-S) in the hearing screening of elderly people who seek for different health care services (audiological clinic and other clinics). Methods: The hearing of 78 elderly -23 attended at the Discipline of Geriatrics and Gerontology of the institution who did not, necessarily, have complaints regarding their hearing, and 55 attended at the Audiology Clinic of the same institution with complaints related to the auditory and/or vestibular systems - was assessed. The HHIE-S questionnaire was applied, and its total score was divided into three categories, according to the handicap perception. Results: A statistically significant relationship was found between handicap and degree of hearing loss in both patients from the Audiology Clinic ( $\mathrm{p}=0.009 *)$ and from the Geriatrics Clinic ( $\mathrm{p}=0.002)$. In the first group, the HHIE-S questionnaire showed low sensitivity $(23.5 \%)$ and high specificity $(73.7 \%)$. In the group of patients from the Geriatrics Clinic, the values of sensitivity (94.7\%) and specificity (75\%) were both high. Conclusions: There was positive association between the degree of hearing loss and the handicap referred by both groups of subjects. The HHIE-S questionnaire is a hearing screening instrument with high sensitivity and specificity in identifying hearing loss in elderly people that seek health care services that are not specific for attention related to hearing disorders.
\end{abstract}

Keywords: Hearing disorders; Hearing loss; Aged; Audiometry; Questionnaires

\title{
REFERÊNCIAS
}

1. Ribeiro A. Aspectos Biológicos do Envelhecimento. In: Russo ICP. Intervenção Fonoaudiológica na Terceira Idade. Rio de Janeiro: Revinter; 1999. p. 1-11.

2. World Health Organization - WHO. International classification of impairments, disabilities and handicaps. Geneva, Switzerland: WHO; 1980.

3. Instituto Brasileiro de Geografia e Estatística - IBGE: Perfil dos idosos responsáveis pelos domicílios no Brasil 2000 [citado 2009 Jul 12].
Disponível em: http://www.ibge.gov.br/home/estatistica/populacao/ perfilidoso/default.shtm

4. Corso JF. Presbycusis, hearing aids and aging. Audiology. 1977;16(2):146-63.

5. Schuknecht HF. Further observations on the pathology of presbycusis. Arch Otolaryngol. 1964 Oct;80:369-82.

6. Hinchcliffe R. The anatomical locus of presbycusis. J Speech Hear Dis. 1962 Nov;27:301-10. 
7. Russo ICP. Distúrbios da audição: a presbiacusia. In: Russo ICP. Intervenção fonoaudiológica na terceira idade. Rio de Janeiro: Revinter; 1999. p. 51-82.

8. Veras RP, Mattos LC. Audiologia do envelhecimento: revisão de literatura e perspectivas atuais. Rev Bras Otorrinolaringol. 2007;73(1):128-34.

9. Ventry IM; Weinstein BE. The Hearing Handicap Inventory for the Eldertly: a new tool. Ear Hear. 1982;3(3):128-34.

10. American Speech-Language-Hearing Association - ASHA. Guidelines for the identification of hearing impairment/ handicap in adult/ elderly persons. ASHA. 1989;31(8):59-63.

11. Davis H. Audiometry: pure tone and simple speech tests. In: Davis H, Silverman SR. Hearind and Deafness. New York: Holt Rinehart and Weston; 1970. p. 179-200.

12. Dalton DS, Cruickchans KJ, Klein BEK, Klein R, Wiley TL, Nondahl DM. The impact of hearing loss on quality of life in older adults. Gerontologist. 2002;43(5):661-68.

13. Sestren E, Jacob LCB, Callefe LG, Alvarenga KF. Avaliaçäo da autopercepçäo do handicap auditivo em idosos. Rev Disturb Comum. 2002;14(1):103-20.

14. Wiley TL, Cruickshanks KJ, Nondahl, DM, Tweed TS. Self-Reported hearing handicap and audiometric measures in older adults. J Am Acad Audiol. 2000;11(2):67-75.
15. Pizan-Faria VM, Iorio MCM. Sensibilidade auditiva e autopercepção do handicap: um estudo em idosos. Disturb Comun. 2004;16(3):289-99.

16. Ventry IM, Weinstein BE. Audiometric correlates of the Hearing Handicap Inventory for the Elderly. J Speech Hear Disord. 1983;48(4):379-84.

17. Nondahl DM, Cruickshanks KJ, Wiley TL, Tweed TS, Klein R; Klein BEK. Accuracy of self-reported hearing loss. Audiology. 1998;27(5):295-301.

18. Gates GA, Murphy M, Rees TS, Fraher A. Screening for handicapping hearing loss in elderly. J Fam Pract. 2003;52(Pt 1):56-62.

19. Nóbrega JD, Câmara MFS, Borges ACC. Audição do idoso: análise da percepção do prejuízo auditivo, perfil audiológico e suas correlações. Rev Bras Prom Saúde. 2008;21(1):39-46.

20. Lichtenstein MJ, Bess FH, Logan AS. Diagnostic performance of Hearing Handicap Inventory for the Elderly (Screening Version) against differing definitions of hearing loss. Ear Hear. 1988;9(4):208-11.

21. Bagai A, Thavendiranathan P, Detsky AS. Does this patient have hearing impairment? Jama. 2006;295(4):416-28.

22. Bess FH, Humes LH. Patologias do sistema auditivo. In: Bess FH, Humes LH. Fundamentos em Audiologia. 2a ed. Porto Alegre: ArtMed; 1998. p. $155-95$. 